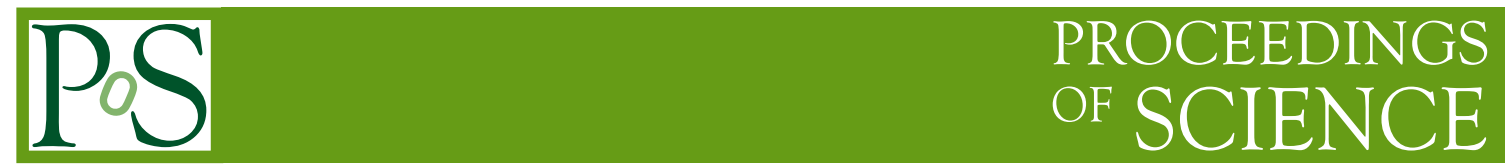

\title{
Inclusive Cross Sections in ME+PS Merging
}

\section{Simon Plätzer*}

Deutsches Elektronen-Synchrotron (DESY), Notkestrasse 85, D-22607 Hamburg, Germany

E-mail: simon.plaetzer@desy.de

We discuss an extension of matrix element plus parton shower merging at leading and nextto-leading order. The algorithm does preserve inclusive cross sections at the respective input order. This constraint avoids potentially large logarithmic contributions, which would require approximate (N)NLO contributions to cancel against.

XXI International Workshop on Deep-Inelastic Scattering and Related Subject-DIS2013, 22-26 April 2013

Marseilles, France

${ }^{*}$ Speaker. 


\section{Introduction}

Parton shower event generators are by now indispensable workhorses of experimental as well as theoretical studies for comparing (standard model) theoretical predictions to measured observables in a most detailed way. Recent years have shown tremendous developments to improve the accuracy of those simulations from the lowest order description of hard scatterings to include exact tree level matrix elements for a large number of jets as pioneered in [1-3], and NLO corrections for the hard scattering itself, [4,5]. Parton shower algorithms themselves have undergone improvements in various directions, see e.g. [6-10] and references therein, which have mainly been driven by easing the combination of parton showers and higher order QCD corrections. Following first steps of combining NLO corrections to the lowest order process while including higher multiplicity tree level matrix elements $[11,12]$, the combination of parton showers and NLO corrections for several jets has been achieved [13-15], partly by including analytic resummation at high logarithmic order [16]. In this contribution, we discuss algorithms to combine parton showers and leading (LO) or next-to-leading order (NLO) calculations for the production of multiple, additional jets, focusing on the accuracy at which inclusive cross sections are reproduced [17-19].

\section{The Landscape of Perturbative QCD}

Jet spectra, as well as event shapes and similar, infrared sensitive observables at colliders exhibit a clear structure of leading contributions at higher orders in the strong coupling. Particularly, the perturbative expansion in terms of the strong coupling $\alpha_{s}$ is typically plagued by large logarithms of the infrared sensitive quantity which eventually overcome the smallness of $\alpha_{s}$ and so render fixed order predictions unreliable. The complications in these regions confront with a certain simplicity of QCD amplitudes which factor in the respective limits into universal and few process dependent pieces at all orders, thus allowing the application of resummation techniques to cure the convergence of the perturbative series.

Notably in QCD (as opposed to electroweak corrections), inclusive quantities are free of large logarithmic corrections with the regions of resolved and unresolved parton emission, or exclusive and inclusive jet cross sections to name an example, delivering equal in magnitude and opposite in sign contributions of large logarithmic enhancement. This situation is illustrated in figure 1, along with an illustration of resummation at next-to-leading logarithmic (NLL) accuracy, taking into account all terms of the form $\alpha_{s}^{n} L^{2 n}$ and $\alpha_{s}^{n} L^{2 n-1}$. Figure 1 also indicates the origin of the different contributions in terms of resolved (emission), and unresolved (virtual), contributions which directly relate to the number of legs and the order in the strong coupling considered.

\section{Parton Showers}

Parton showers simulate the multiple emission of soft and collinear partons originating from a hard process configuration $\phi_{n}$ driven by a differential cross section $\mathrm{d} \sigma\left(\phi_{n}, q_{n}|\cdots| q_{0}\right)^{1}$. The sequence of scales $q_{n}<q_{n-1}, \ldots, q_{0}$ is either directly determined from shower evolution, or is assigned by a clustering procedure corresponding to the inverse of a possible shower evolution $\phi_{0} \rightarrow \phi_{n}$, if

\footnotetext{
${ }^{1}$ See [17] for details on the notation
} 

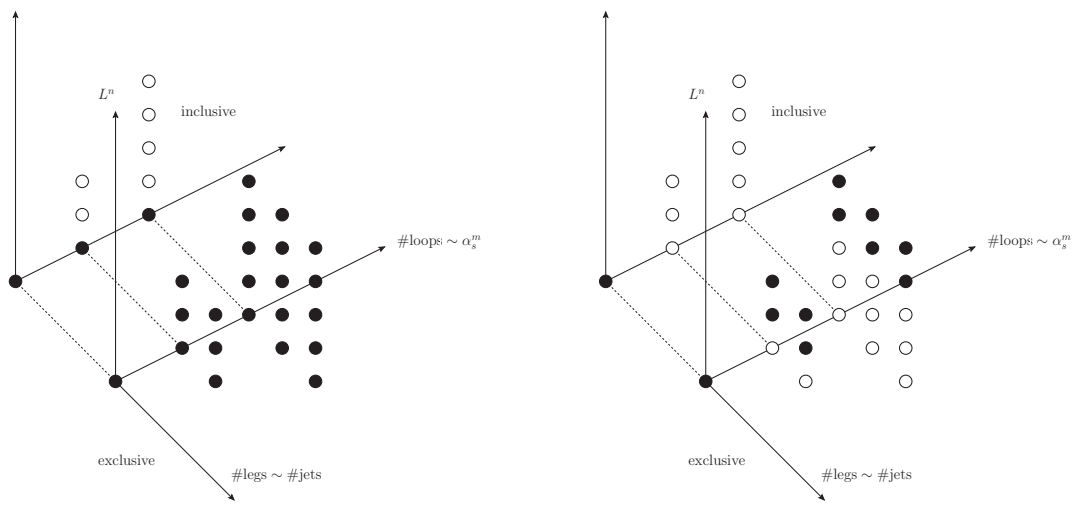

Figure 1: Illustration of leading contributions to infrared sensitive observables in QCD perturbation theory. At each order in the strong coupling, large logarithmic contributions appear which cancel in inclusive quantities (left panel). The vertical axis indicates powers of large logarithms in dependence of the order in the strong coupling and the number of resolved or unresolved parton emissions (horizontal axes). Resummation at a certain logarithmic accuracy takes sums parts of these contributions to all orders (left panel, exemplifying a NLL resummation).

the cross section is determined by exact matrix elements. Sensible parton shower algorithms can up to date only be proven to perform resummation at the level of leading logarithms, but there are strong indications that a large part of the next-to-leading logarithmic contributions are present, at least in special cases. Parton showering acts on cross sections driving events $\phi_{n}$ as

$$
\mathrm{PS}_{\mu}\left[\mathrm{d} \sigma\left(\phi_{n}, q_{n}\right)\right]=\mathrm{d} \sigma\left(\phi_{n}, q_{n}\right) \Delta_{n}\left(\mu \mid q_{n}\right)+\mathrm{PS}_{\mu}\left[\mathrm{d} \sigma\left(\phi_{n}, q_{n}\right) \frac{\mathrm{d} \phi_{n+1}}{\mathrm{~d} \phi_{n}} P_{\mu}\left(\phi_{n}, q_{n+1}\right) \Delta_{n}\left(q_{n+1} \mid q_{n}\right)\right]
$$

where

$$
\Delta_{n}(q \mid Q)=\exp \left(-\int_{q}^{Q} \mathrm{~d} k \frac{\mathrm{d} \phi_{n+1}}{\mathrm{~d} \phi_{n} \mathrm{~d} k} P\left(\phi_{n}, k\right)\right),
$$

is the Sudakov form factor which can be interpreted as the probability for no emission between a hard scale $Q$ and a soft scale $q$. The cancellation of large logarithmic contributions in inclusive quantities is inherent to parton shower evolution by the fact that this evolution is a stochastic process being subject to unitarity constraints,

$$
\int_{q}^{q_{k-1}} \mathrm{~d} q_{k} \frac{\mathrm{d} \phi_{k}}{\mathrm{~d} \phi_{k-1} \mathrm{~d} q_{k}} P\left(\phi_{k-1}, q_{k}\right) \Delta_{k-1}\left(q_{k} \mid q_{k-1}\right)=1-\Delta_{k-1}\left(q \mid q_{k-1}\right) .
$$

Considering inclusive and exclusive cross sections of emitting at least $n$ or exactly $n$ resolved partons, parton shower evolution will generate the cross sections

$$
\begin{array}{ll}
=n & \mathrm{~d} \sigma^{(0)}\left(\phi_{0}, q_{0}\right) \frac{\mathrm{d} \phi_{n}}{\mathrm{~d} \phi_{0}} P_{\mu}\left(\phi_{n-1}, q_{n}\right) \cdots P_{\mu}\left(\phi_{0}, q_{1}\right) \Delta_{n}\left(\mu\left|q_{n}\right| \cdots \mid q_{0}\right) \\
\geq n & \mathrm{~d} \sigma^{(0)}\left(\phi_{0}, q_{0}\right) \frac{\mathrm{d} \phi_{n}}{\mathrm{~d} \phi_{0}} P_{\mu}\left(\phi_{n-1}, q_{n}\right) \cdots P_{\mu}\left(\phi_{0}, q_{1}\right) \Delta_{n-1}\left(q_{n}|\cdots| q_{0}\right) .
\end{array}
$$

We will take this observation as a starting point to constrain inclusive cross sections when combining parton showers and higher multiplicity jet cross sections at leading order (LO) and next-toleading order (NLO). 

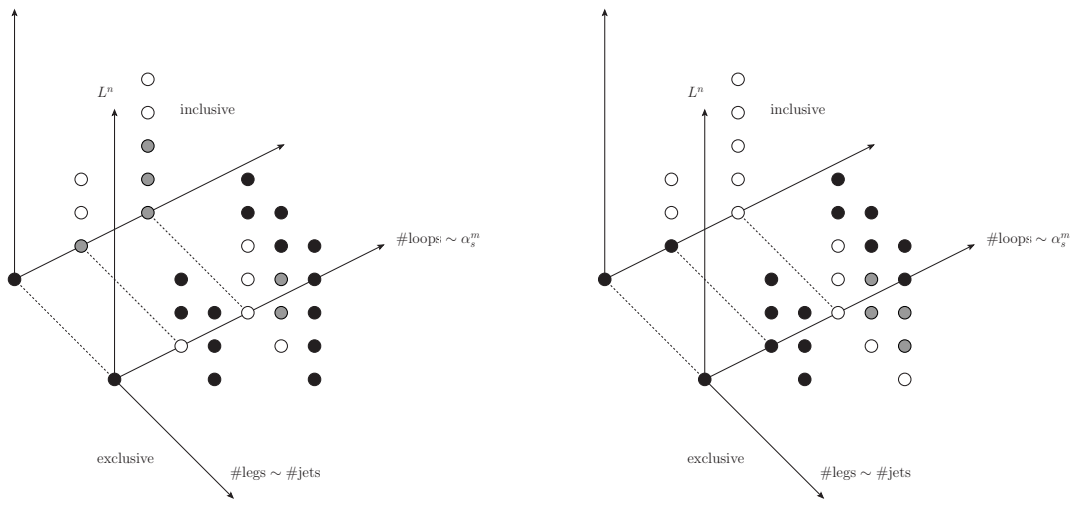

Figure 2: Merging tree level matrix elements and parton showers (left panel) allows to the exact description of subleading logarithmic contributions stemming from resolved, tree level contributions (up to two additional parton emissions in the case considered here). This will yield a partially improved description of contributions which would only be accessible from NLO calculations and may leave its traces in inclusive quantities (see text for details). This is to be confronted with NLO matching (right panel), which includes tree level contributions for one additional parton emission, as well as the exact one-loop pieces without modifying the inclusive cross section except adding the first order correction to it.

\section{Tree Level Merging Revisited}

The merging of tree level matrix elements and parton showers aims at improving the description of multiple parton emission by the respective, exact, tree level matrix elements as far as computationally feasible. This may either be done across all of phase space or only in a certain region for large-angle, hard emissions. Generically, we impose a merging condition to replace the product of lowest order cross section and parton shower splitting probabilities by their exact counterpart in exclusive cross sections generated by the merging algorithm, while keeping the Sudakov form factor accounting for exclusiveness of the respective final state. Assuming the presence of tree level matrix elements for emission of up to $N$ additional partons, and introducing a 'hard' region as resolved by a merging cutoff $\rho$ above the shower cutoff $\mu$, we have

$$
\begin{aligned}
& \mathrm{PS}_{\mu}\left[\mathrm{d} \sigma_{N, \rho}^{\text {merged }}\right]= \\
& \quad \operatorname{PS}_{\mu \mid \rho}\left[\sum_{k=0}^{N-1} \mathrm{~d} \sigma_{\rho}^{(0)}\left(\phi_{k}, q_{k}\right) \Delta_{k}\left(\rho\left|q_{k}\right| \cdots \mid q_{0}\right)+\mathrm{PS}_{\rho}\left[\mathrm{d} \sigma_{\rho}^{(0)}\left(\phi_{N}, q_{N}\right) \Delta_{N-1}\left(q_{N}|\cdots| q_{0}\right)\right]\right],
\end{aligned}
$$

i.e. shower emissions are restricted to a range between the merging scale $\rho$ and the infrared cutoff $\mu$ (as indicated by the symbol $\mathrm{PS}_{\mu \mid \rho}$ ) for all but the highest matrix element multiplicity available, and otherwise unconstrained. The general solution to this condition is given in [17], and involves subtraction of the parton shower approximation in the hard region above $\rho$, accompanied by an appropriate Sudakov reweighting. This is illustrated in figure 2 and compared to NLO matching.

\section{Inclusive Cross Sections as a Guide to NLO Merging}

By definition of the tree level merging condition, exclusive cross sections resemble the func- 
tional form expected from parton shower evolution; since no modification of the Sudakov form factors has been made to restore the unitarity of the merging algorithm, inclusive cross sections do, however, not reflect the expected pattern except for the highest matrix element multiplicity available. Starting from the next-to-maximal multiplicity, we instead find for the $n-1$ parton inclusive cross section:

$$
\begin{aligned}
& \mathrm{d} \sigma_{\rho}^{(0)}\left(\phi_{n-1}, q_{n-1}\right) \Delta_{n-2}\left(q_{n-1}|\cdots| q_{0}\right)+ \\
& \quad \int_{\rho}^{q_{n-1}} \mathrm{~d} q_{n}\left(\frac{\mathrm{d} \sigma_{\rho}^{(0)}\left(\phi_{n}, q_{n}\right)}{\mathrm{d} q_{n}}-\frac{\mathrm{d} \phi_{n}}{\mathrm{~d} \phi_{n-1} \mathrm{~d} q_{n}} P_{\rho}\left(\phi_{n-1}, q_{n}\right) \mathrm{d} \sigma_{\rho}^{(0)}\left(\phi_{n-1}, q_{n-1}\right)\right) \Delta_{n-1}\left(q_{n}|\cdots| q_{0}\right) .
\end{aligned}
$$

The first term is the expected result, while the correction to it involves an integral over the difference between an $n$ parton cross section and the approximation to it when replacing the softest emission to be driven by the parton shower. Provided that the parton shower is a reasonable approximation to the singly unresolved behavior of tree level matrix elements, this contribution will not contribute logarithmically enhanced contributions to inclusive cross sections. The situation for merging parton showers and NLO corrections of different jet multiplicity will be different. Here, the tree level (i.e. LO) exclusive $n$-parton cross sections (according to the shower resolution with resolution parameter $\rho$ ) will be replaced by their NLO counter parts as achieved e.g. by the algorithms outlined in [13-15], while the shower kernels still reflect the unresolved behavior of tree level matrix elements, thus contributing higher logarithmic order terms to inclusive quantities.

As a first step towards NLO merging with inclusive cross sections which are not out of control, we therefore attempt to restore the LO inclusive cross sections within a tree level merging algorithm, first. Having identified the contribution by which inclusive cross sections are spoiled, it is straightforward to subtract these contributions from the merged cross section in an algorithmic way: Within the merged cross section, for all but the highest multiplicity, the integral over the phase space for the softest emission in the $n+1$ parton contribution needs to be subtracted from the $n$ parton contribution. This algorithm amounts to a variant of thew LoopSim method [20] and in the case of tree level merging does generate approximate one-loop contributions, thus rendering the LO merging effectively an nLO merging.

Expanding exclusive $n$-parton cross sections above the merging scale $\rho$ to first order in the strong coupling, one readily identifies the correction to promote the nLO cross section to a full NLO cross section. The correction is given by the NLO calculation differential in the Born variables, which will otherwise be treated in the same way as the tree level input to the merging algorithm. The problematic contributions in inclusive cross sections, as discussed previously, are now manifest. We can, however, add correction terms similar to the ones used for tree level merging to restore the exact NLO inclusive cross section times the appropriate Sudakov suppression in a similar algorithm. This procedure will generate approximate two loop contribution, effectively rendering the algorithm a merging of LoopSim nNLO calculations.

\section{Conclusions and Outlook}

We have presented an extension to multileg matrix element and parton shower merging, which preserves inclusive cross sections at the level of the available accuracy, particularly at tree and 
one-loop level. This constraint is of utmost importance particularly for the latter case, as NLO corrections to shower splitting kernels are so far out of reach. The procedure starts from merging LO calculation of different jet multiplicities, will generate approximate NLO corrections similar to the LoopSim algorithm when imposing constraints on inclusive cross sections and thus serves as a guide to NLO merging. Repeating the procedure in this case yields approximate NNLO corrections and we anticipate that this could well serve as a guide to NNLO plus parton shower matching. An implementation of this algorithm based on the Herwig++ Matchbox framework [8] is underway.

\section{Acknowledgments}

This work was supported by the Helmholtz Alliance "Physics at the Terascale".

\section{References}

[1] S. Catani, F. Krauss, R. Kuhn, and B. R. Webber, QCD Matrix Elements + Parton Showers, JHEP 11 (2001) 063, [hep-ph/0109231].

[2] L. Lönnblad, Correcting the colour-dipole cascade model with fixed order matrix elements, JHEP 05 (2002) 046, [hep-ph/ 0112284$].$

[3] F. Krauss, Matrix elements and parton showers in hadronic interactions, JHEP 08 (2002) 015, [hep-ph/0205283].

[4] S. Frixione and B. R. Webber, Matching NLO QCD computations and parton shower simulations, JHEP 06 (2002) 029, [hep-ph/ 0204244$].$

[5] P. Nason, A new method for combining NLO QCD with shower Monte Carlo algorithms, JHEP 11 (2004) 040, [hep-ph/ 0409146$].$

[6] S. Schumann and F. Krauss, A Parton shower algorithm based on Catani-Seymour dipole factorisation, JHEP 03 (2008) 038, [arXiv: 0709 . 1027].

[7] W. T. Giele, D. A. Kosower, and P. Z. Skands, A Simple shower and matching algorithm, Phys. Rev. D78 (2008) 014026, [arXiv: 0707.3652$].$

[8] S. Platzer and S. Gieseke, Dipole Showers and Automated NLO Matching in Herwig++, Eur.Phys.J. C72 (2012) 2187, [arXiv:1109.6256].

[9] W. Kilian, J. Reuter, S. Schmidt, and D. Wiesler, An Analytic Initial-State Parton Shower, JHEP 1204 (2012) 013, [arXiv:1112.1039].

[10] S. Platzer and M. Sjodahl, Subleading Nc improved Parton Showers, JHEP 1207 (2012) 042, [arXiv:1201.0260].

[11] K. Hamilton and P. Nason, Improving NLO-parton shower matched simulations with higher order matrix elements, JHEP 1006 (2010) 039, [arXiv: 1004 . 1764].

[12] S. Hoche, F. Krauss, M. Schonherr, and F. Siegert, NLO matrix elements and truncated showers, arXiv:1009.1127.

[13] N. Lavesson and L. Lonnblad, Extending CKKW-merging to One-Loop Matrix Elements, JHEP 12 (2008) 070, [arXiv:0811.2912].

[14] S. Hoeche, F. Krauss, M. Schonherr, and F. Siegert, QCD matrix elements + parton showers: The NLO case, arXiv:1207.5030. 
[15] R. Frederix and S. Frixione, Merging meets matching in MC@NLO, arXiv:1209.6215.

[16] S. Alioli, C. W. Bauer, C. J. Berggren, A. Hornig, F. J. Tackmann, et. al., Combining Higher-Order Resummation with Multiple NLO Calculations and Parton Showers in GENEVA, arXiv:1211.7049.

[17] S. Platzer, Controlling inclusive cross sections in parton shower + matrix element merging, arXiv: 1211.5467.

[18] L. Lonnblad and S. Prestel, Unitarising Matrix Element + Parton Shower merging, arXiv:1211.4827.

[19] L. Lonnblad and S. Prestel, Merging Multi-leg NLO Matrix Elements with Parton Showers, arXiv:1211.7278.

[20] M. Rubin, G. P. Salam, and S. Sapeta, Giant QCD K-factors beyond NLO, JHEP 1009 (2010) 084, [arXiv:1006.2144]. 